\title{
Micro-chemical analysis of highly heat loaded CFC/Cu interfaces from Tore Supra and Wendelstein 7-X
}

\author{
T. Höschen ${ }^{1,}$, Ch. Linsmeier ${ }^{1}$, H. Greuner ${ }^{1}$, M. Missirlian ${ }^{2}$ \\ ${ }^{1}$ Max-Planck-Institut für Plasmaphysik, EURATOM Association, \\ Boltzmannstr. 2, 85748 Garching b. München, Germany \\ ${ }^{2}$ CEA, IRFM, 13108 Saint-Paul-Lez-Durance, France
}

\begin{abstract}
A comparison of high heat flux (HHF) tested $\mathrm{CFC} / \mathrm{Cu}$ materials of Tore Supra and Wendelstein 7-X plasma-facing components is performed in order to understand the different fatigue behavior of the bonding interface, in particular for the Tore Supra materials. The elemental distribution around the bonding layer and the chemical composition of the active element titanium are characterized by secondary ion mass spectrometry (SIMS) and X-ray photoelectron spectroscopy (XPS). The results show that the improved bonding of the Wendelstein 7-X target elements compared to the Tore Supra pump limiter elements is due to a modified silicon and titanium distribution at the bonding interface. However, the difference of the fatigue behavior between the two Tore supra components cannot be attributed to the bonding interface, since the elemental distribution and chemistry for these components are identical and no degradation is observed after extended heat flux exposure.
\end{abstract}

\section{Introduction}

For the toroidal pump limiter (TPL) elements of Tore Supra (TS), two different qualities of carbon-fiber reinforced carbon (CFC) material, produced by SNECMA, were applied [1]. While SEP N11-92 (produced in 1992) was within the specifications, the N11-98 (produced in 1998) showed a slight deviation in its mechanical properties. Small differences in the percentage range were measured for the density, tensile and shear stress [6]. After both batches were finally accepted for production, the so-called "TS finger elements" equipped with N11-98 tiles showed a much higher failure rate and degraded performance. For Wendelstein 7-X (W7-X), a similar but further developed CFC material (NB31) from the same manufacturer is used at the target elements. Critical to the target elements is the bonding between the CFC as plasma-facing material and the Cu-based heat sink structure. Over the years, the active metal casting (AMC) process and the laser structuring of the CFC material have been optimized by PLANSEE SE. The advanced AMC is based on a Ti alloy with an addition of $\mathrm{Si}$ [5] acting as bond between the CFC and $\mathrm{Cu}$ structures.

The degradation of performance, as observed for the TS target elements with different CFC batches, has only recently been investigated in high heat flux tests [1]. In this study, we focus on the properties of the AMC bonding layer. Samples from the different TPL and W7-X target elements were exposed to high heat fluxes and subsequent elemental and chemical analysis of the bonding layer is applied to study a possible degradation of this interface as a potential failure mechanism. The TS target elements with different CFC material and a CFC NB 31-Cu interface of a pre-series target for the W7-X divertor are analyzed under the same conditions.

\section{Investigated samples}

The samples under investigation were prepared from two TPL finger elements using N11 CFC from the different production batches. S390 is covered with the N11-98 CFC material, S703 is covered with the N11-92 CFC material. They were never installed in the TS tokamak. 
The high heat flux (HHF) tests were performed in their as-produced state at the neutral beam facility GLADIS at IPP [2]. The GLADIS facility is equipped with two ion sources, each 1 MW power. The tests were performed with a $30 \mathrm{keV}$ hydrogen beam. The Gaussian shaped neutral beam generates $80-100 \%$ of its nominal heat flux in a diameter of $75 \mathrm{~mm}$ at the target position. The elements were installed in parallel in the test chamber. The water cooling conditions were similar to the TS cooling conditions, except that the water temperature is $20^{\circ} \mathrm{C}$ in GLADIS. To achieve the same CFC/Cu interface temperature as for $8 \mathrm{MW} / \mathrm{m}^{2}$ heat flux and $120^{\circ} \mathrm{C}$ coolant temperature in TS, it was necessary to increase the applied heat flux to $10 \mathrm{MW} / \mathrm{m}^{2}$ in GLADIS. Both elements were heat loaded simultaneously with 200 pulses at $10 \mathrm{MW} / \mathrm{m}^{2}$, $10 \mathrm{~s}$ pulse length, and additional 3000 pulses of $8 \mathrm{MW} / \mathrm{m}^{2}$. While the $\mathrm{S} 703$ element remained undamaged, the two tiles 12 and 17 on the S390 element failed during the HHF test. In Figure 1 an infrared image of the TS samples during HHF loading in GLADIS is shown. In this image, the central parts (green to yellow) represent the center of the neutral beam. The bright colors (red to white) represent the positions of tiles 12 and 17, which were removed after failure of the bonding interface during earlier cycles. For detailed investigations of the CFC-Cu interfaces, from both elements S703 and S390 the undamaged tiles 16 were chosen for the analysis described in the following sections. The interfaces of the TS elements are compared to a similarly prepared W7-X pre-series III target. The element 4S-045 was loaded in GLADIS with 5000 pulses of $10 \mathrm{MW} / \mathrm{m}^{2}$ each. No defects were observed through overheated tiles at this element, although even higher heat loads were applied.

\section{Results and Discussion}

\subsection{Characterization of the $\mathrm{CFC}-\mathrm{Cu}$ interface}

From the target elements, samples are prepared as metallographic cross sections by cutting and polishing. Micrographs representing the three different elements are shown in Figures 2, 3 , and 4. For the TS materials, the top part in the figures shows an overview and a detail section, marked by the rectangle in the overview image. The square in the detail images represents the area in which secondary ion mass spectrometry (SIMS) analysis is performed. $\mathrm{X}$-ray photoelectron spectroscopy (XPS) analysis is performed within this square region, whereby the XPS analysis spot is centered at the bonding interface. After optical micrography, the samples were introduced in a multi-analysis vacuum system capable of performing XPS and SIMS. In addition, the device is equipped with a scanning electron microscope assisting investigations by producing secondary electron (SE) pictures.

To investigate the chemical state of the elements at the interface between the CFC and the $\mathrm{Cu}$ heat sink, XPS measurements are performed. Adsorbed contaminations from the air are removed using a $5 \mathrm{kV} \mathrm{Ar}{ }^{+}$ion beam for $20 \mathrm{~s}$ on an area of $1 \times 1 \mathrm{~mm}^{2}$, applying a fluence of approx. $1 \times 10^{16} \mathrm{~cm}^{-2}$. The analyzed area from which photoelectrons are detected is set to $400 \mu \mathrm{m}$, despite the smaller width of the interface zone (less than $100 \mu \mathrm{m}$, as can be seen e.g. in Figure 4). Since Ti is only present in this bonding layer (details see below in the next Section), the signal for this element is detected selectively from the bonding area. As a consequence, the $\mathrm{Ti}$ chemical state measured by XPS represents selectively the interface chemistry. The $\mathrm{C}$ signal, however, is averaged over both the interface area and the CFC matrix. Therefore, the detected $C$ chemical states are representative for $C$ in the CFC as well as for $\mathrm{C}$ in the bonding layer.

For SIMS measurements, a primary ion beam of $15 \mathrm{kV} \mathrm{O}^{-}$ions was focused to a spot size of about $\sim 50 \mu \mathrm{m}$ with a current of $93 \mathrm{nA}$. The focused beam of primary ions hits the sample and removes atoms from the surface. A few ( 1\%) of the sputtered atoms leave the surface ionized and the fraction that enters the entrance of a quadrupole mass spectrometer can be analyzed due to their mass-to-charge ratio. The primary beam is scanned over the samples 
surface and thus allows the construction of a 2D elemental map point by point. After previous implantation of oxygen to raise and stabilize the secondary ion yield an area of approximately $1 \times 1 \mathrm{~mm}^{2}$ was measured with a scanning field of 128x128 pixels, $40 \mathrm{~s} / \mathrm{pixel} / \mathrm{mass}$ dwell time.

\subsection{Chemical state of the interface elements: XPS results}

The AMC process involves $\mathrm{Ti}$ as an active element in the bonding between $\mathrm{CFC}$ and $\mathrm{Cu}$. $\mathrm{Ti}$ can form alloys with $\mathrm{Cu}$ and carbides with $\mathrm{C}$. Therefore, the chemical state of the Ti present in the bonding layer indicates whether the bonding process is complete. In XPS spectra a shift of the photoelectron signal indicates a chemical reaction. The spectra are measured using Al $\mathrm{K} \alpha$ radiation in order to avoid an overlap of the Cu KLL Auger peaks with the Ti $2 p$ region. In Figure 5, XPS spectra in the Ti $2 p$ and $\mathrm{C} 1 s$ regions are shown for the three different target elements. In all cases, the Ti $2 p_{3 / 2}$ peak is located at a binding energy of $454.6 \mathrm{eV}$, which is characteristic for titanium carbides [3]. This demonstrates that the Ti present at the bonding interface has reacted with the CFC to form TiC. This reaction is confirmed by a peak around $282 \mathrm{eV}$ in the C 1s spectra, also shown in Figure 5. However, since the interface layer represents only a small part of the analysis area, the carbide signal represents $1.5-4 \%$ of the total C $1 s$ intensity. Nevertheless, the carbide peak can be identified unambiguously by peak fitting. The fit components are overlaid to the measured data in the plots. The elemental carbon in the CFC is represented by the two dominating peaks above 284 and $285 \mathrm{eV}$. The carbide is formed already during the bonding process, as could be shown earlier in the comparison of an unloaded W7-X target element with an element after extended high heat loading [4].

\subsection{Elemental distribution at the interface: SIMS results}

The 2D elemental distribution across the bonding layer is measured by SIMS. The results for the elements $\mathrm{C}, \mathrm{Cu}, \mathrm{Ti}$, and $\mathrm{Si}$, together with a secondary electron image of the investigated areas, are displayed in Figure 6. For each sample the results are presented in a separate column, in the horizontal rows the respective elements for the three materials are compared. Due to chemical matrix effects, SIMS is not an absolutely quantitative technique. The secondary ion yield for different elements can vary over orders of magnitude under the same measurement conditions. Only extensive studies on known standards allow the assignment of absolute concentrations, which were not performed in this work. Nevertheless, since the chemical composition of the samples under comparison is very similar, the intensities for each element can be compared relative to the other samples. In the 2D images, the intensity is color coded and the scale is identical for one element of all three samples (shown to the right).

The secondary electron (SE) picture of each investigated region is presented in the first row. The SE pictures are taken directly after the SIMS measurements. Therefore, the area sputtered and analyzed during the SIMS measurement is visible as a square with slightly different secondary electron yield, only barely visible in the rightmost picture of the W7-X sample 4S-045. Together with the respective optical micrographs (Figures 2-4), they provide information on the samples topography. Preparation of porous CFC material often results in coarse microsections. Whereas in the optical micrographs the pores appear dark as they are out of the focal plane, in the SE picture the topography appears due to different secondary electron yield on pores and edges. The laser structured CFC surface in the upper part of the images and the respective metallic needles from the $\mathrm{Cu}$ heat sink below are clearly visible in Fig. 3. A comparison of Fig. 3 and Fig.4 illustrates the improvement of the laser structuring process applied on the W7-X CFC.

The SIMS 2D distribution of $\mathrm{C}$ is displayed in the second row. Since the samples are measured under identical conditions, the color-coded intensity is comparable between the 
three samples. The carbon-dominated areas in the upper part of the images all exhibit a smooth blue color. This indicates that these polished CFC areas have the same surface quality, as their $\mathrm{C}$ secondary ion yield is similar. This is an expected result and a confirmation for the comparability of the three measurements. The two TS samples S390 and S703 show higher count rates at positions where the carbon ions originate from the rough surface inside CFC-pores near the carbon-copper interface. This does not mean that there is a higher amount of carbon, but the roughness enhances the sputtering yield and thus the number of secondary ions measured. The lower intensity and less peaks for the W7-X 4S-045 sample indicates a better polished surface, which is confirmed by Fig. 4 .

The 2D maps for $\mathrm{Cu}$ are complementary to the $\mathrm{C}$ measurements. Also here, the well polished areas show a smooth count rate distribution. Higher signal intensity is in this case received mainly in the W7-X 4S-045 picture from the border seam at the copper cones. The constant width of the bright line around this structure of approximately the width of the incident ion beam $(50 \mu \mathrm{m})$ indicates that this is again a topographic effect. Introduced by the metallographic preparation, a little step between copper and the recessed carbon surface leads to an increased $\mathrm{Cu}$ sputtering yield at the edge. Whenever a part of the primary beam hits this edge, a high fraction of $\mathrm{Cu}$ is eroded at the seam, resulting in the bright line around the copper cone in the SIMS map of the W7-X 4S-045 sample. This effect is not as pronounced for the TS samples. Nevertheless, in all cases a sharp interface between CFC and $\mathrm{Cu}$ is observed.

In the next two rows, the $2 \mathrm{D}$ distribution of $\mathrm{Ti}$ and $\mathrm{Si}$ is shown. For these two elements, a strong difference between the earlier TS components and the newer W7-X target element can be seen. The Ti maps demonstrate one of the improvements made through the refinement of the AMC process, as described in [5]. Only in the newer W7-X 4S-045 sample there is a continuous $\mathrm{Ti}$ signal along the $\mathrm{C}-\mathrm{Cu}$ interface, indicating that $\mathrm{Ti}$ is distributed evenly at the bonding interface during the AMC process. In both TS samples the distribution of titanium appears irregular along the interface. In the TS samples, Ti is present only in the dips drilled in the CFC or, to a smaller amount, in the CFC pores. Between the two TS materials, however, no difference in the Ti distribution is observed. The apparent higher Ti amount for TS S703 in the shown images is not significant, as the analysis of a number of interface areas demonstrates.

Another improvement of the AMC process between the TS and W7-X components appears in the Si maps. While for the TS samples Si is present mostly in the CFC region as an impurity, in the W7-X 4S-045 sample the silicon signal can be seen clearly in the AMC regions together with the Ti signal. For the TS samples, the areas with high Ti intensities have only little or no Si signal. The regions where the AMC alloy is present are recognized well also in the SE images, as they exhibit a different secondary electron yield compared to the neighboring copper. They appear a little darker. Thus, the addition of Si to the AMC alloy is visualized. The reasons for an admixture of Si to the AMC alloy are described in [5]. The improved AMC bonding region is best visible in the W7-X 4S-045 sample, more precisely in the left of these two cones. At the tip of this cone the sample surface cuts through the AMC layer, resulting in a large bonding layer area in the 2D maps. Intensity from all elements is detected in this region. It most strongly shows up in the titanium map, but also contains copper, silicon and carbon as a result of the carbide formation, on which the AMC mechanism is based.

\section{Conclusions}

Since neither the elemental distribution (as measured by SIMS), nor the carbide formation at the bonding interface (as measured by XPS) differ between the two TS materials, the AMC 
bonding layer can be excluded as reason for the failure of the S390 component. In all samples titanium is present exclusively in a carbide bonding state. This allows the wetting with copper and facilitates the CFC-Cu bonding. The titanium amount along the interface appears to be low and unevenly distributed in both TS cases, whereas the W7-X 4S-045 sample shows an improved Ti distribution as well as Si within the bonding layer. Si is completely missing in the Ti-containing areas of both TS materials. Since no difference could be revealed between the two TS bonding layers, the reason for the different failure behavior has to be attributed to the differences in the properties of the CFC grades.

\section{References}

[1] M. Missirlian, H. Greuner, T. Höschen, Ch. Linsmeier, M. Richou, M. Lipa, B. Böswirth, J. Boscary, Fusion Eng. Des. (2011), doi:10.1016/j.fusengdes.2011.03.113

[2] H. Greuner, B. Böswirth, J. Boscary, P. McNeely, J. Nucl. Mat. 367-370 (2007) 1444

[3] J. Luthin and Ch. Linsmeier, Phys. Scr. T91 (2001) 134.

[4] H. Greuner, B. Böswirth, J. Boscary, T. Friedrich, C. Lavergne, Ch. Linsmeier, J. Schlosser and A. Wiltner, Fusion Eng. Des. 84 (2009) 848.

[5] E. Eidenberger, C. Scheu, G. Hawranek, R. Pippan, B. Schedler, H. Leitner and H. Clemens, Adv. Eng. Mater. 8 (2006) 1092

[6] M. Lipa, et al., Fusion Science and Technology 56 Part 3 (2009) 1124-1149 


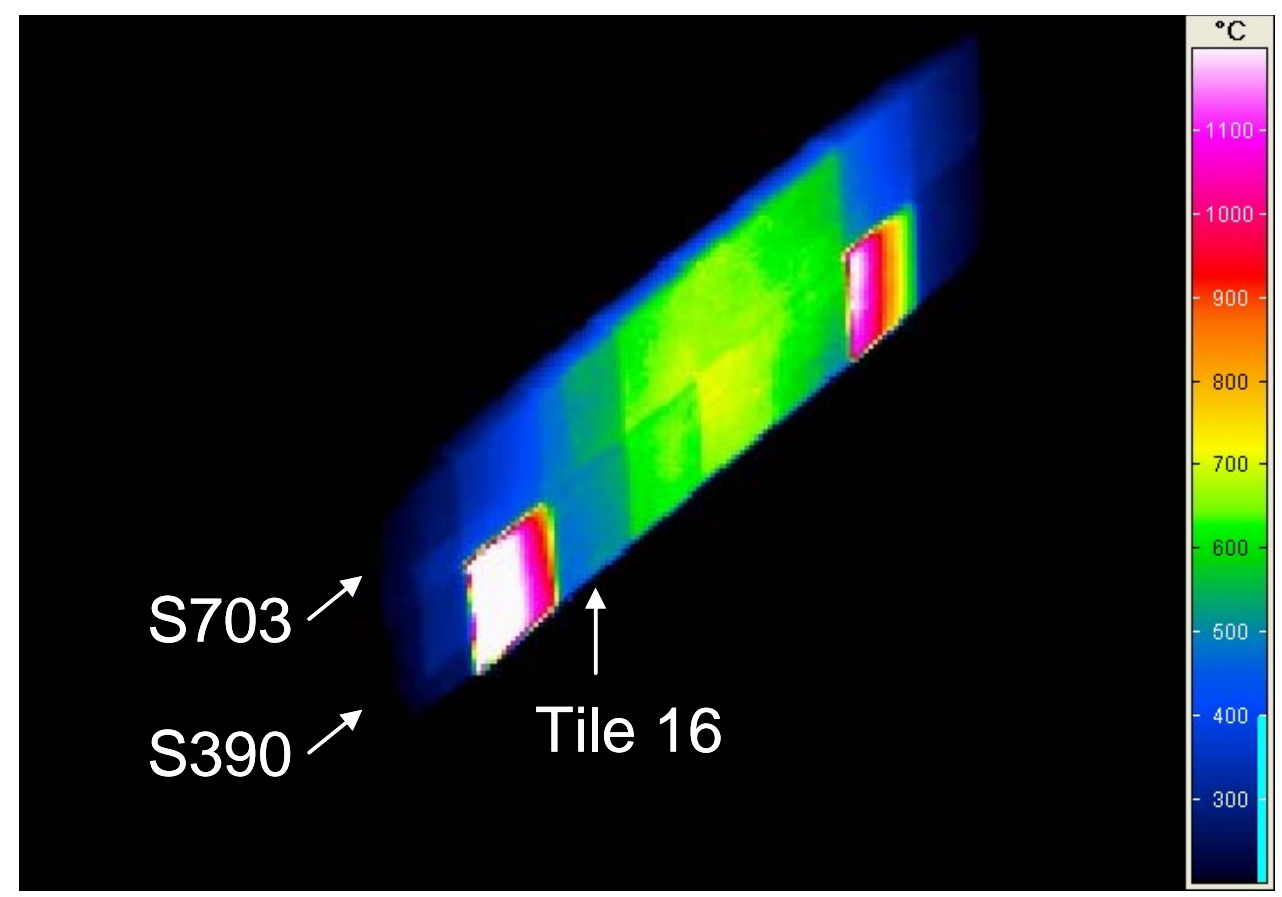

Figure 1 Color-coded infrared image of the two TS finger elements tested simultaneously in GLADIS. Tiles 12 and 17 are already removed from the S390 component. The clearly visible hot spots correspond to remaining CFC/AMC interface of the removed tiles 


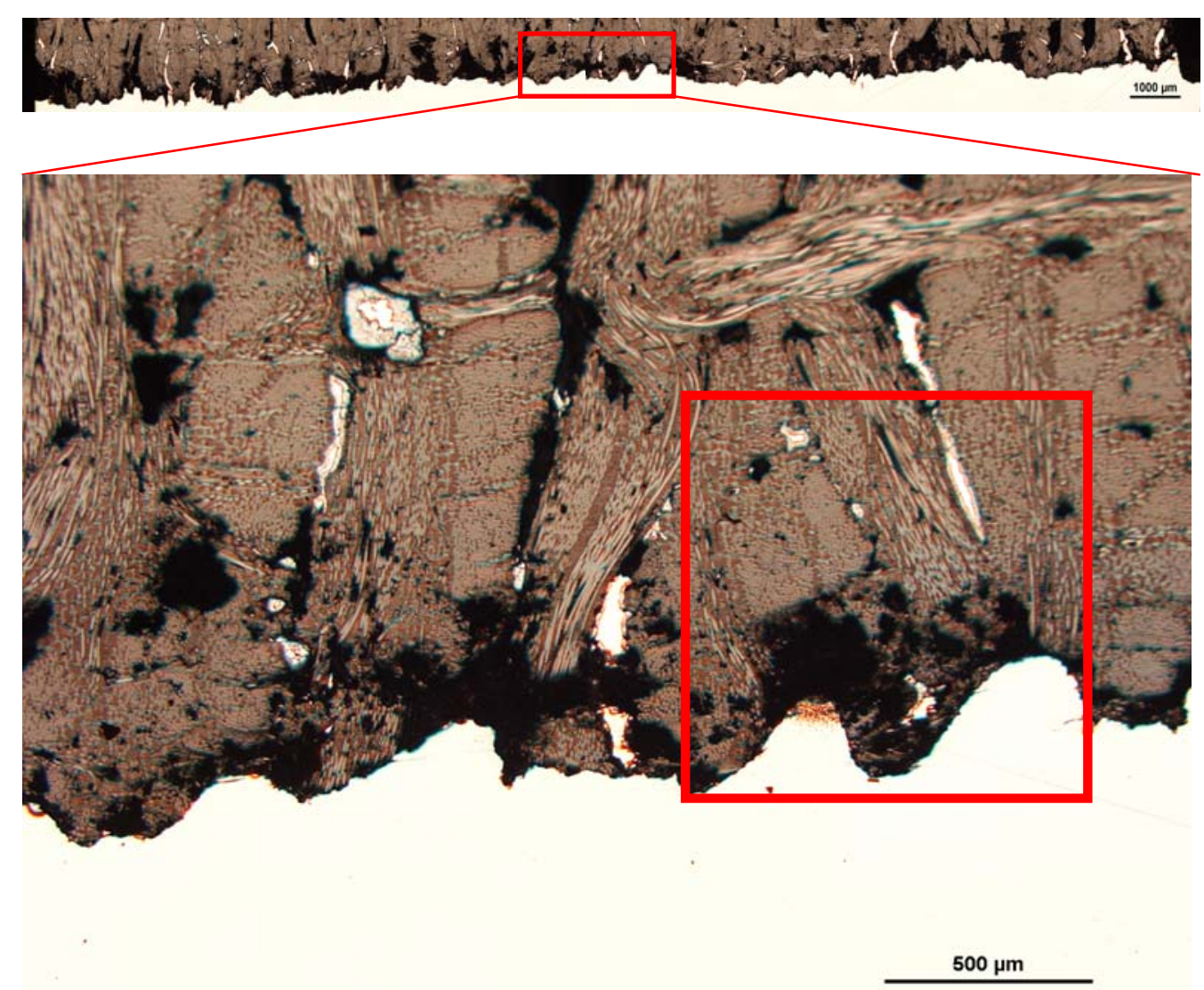

\section{1)}

Figure 2 Optical micrographs of the CFC-Cu interface region on TS S390 tile 16 after metallographic preparation. The marked area in the larger detail section represents the area analyzed by SIMS. 

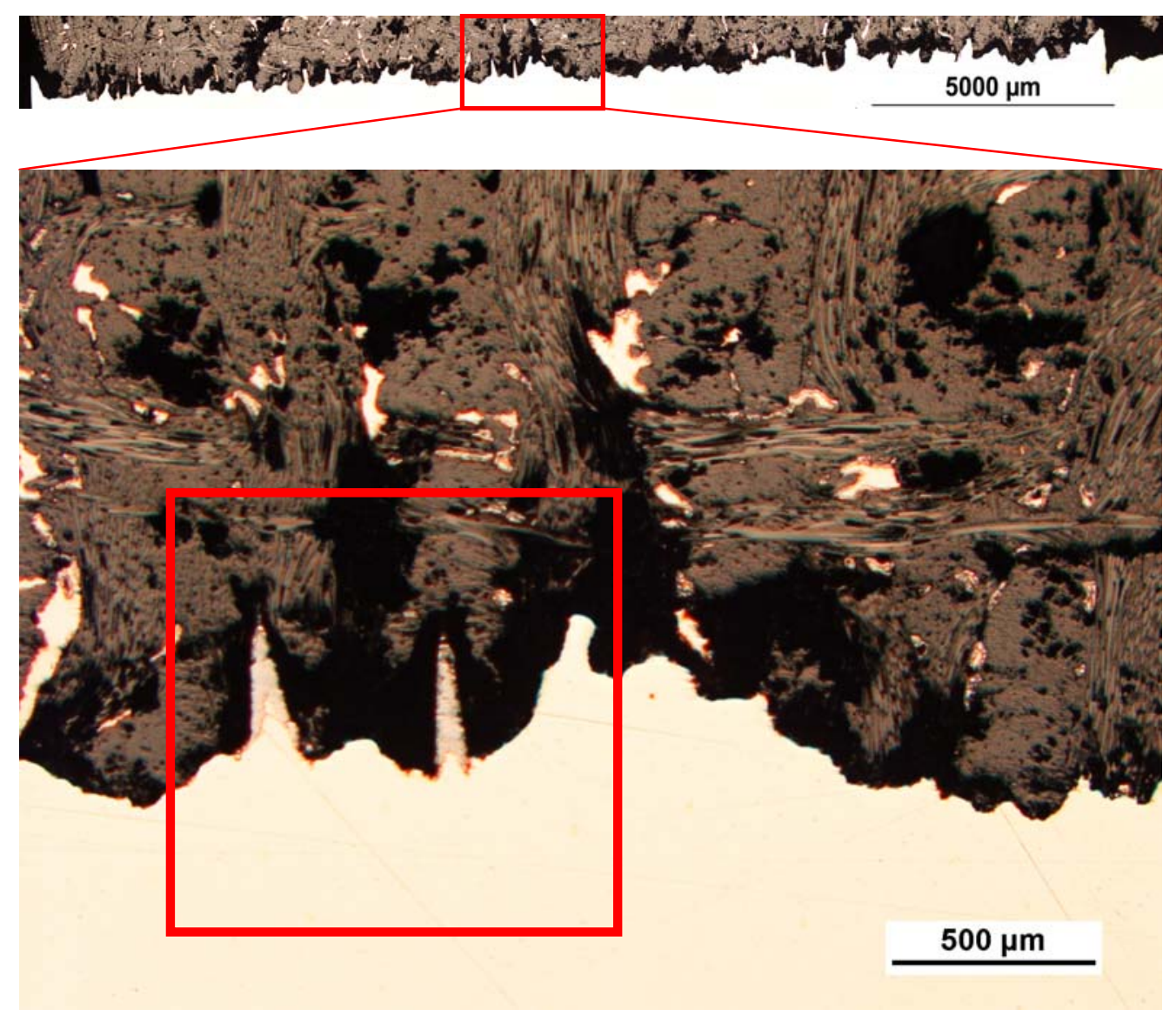

Figure 3 Optical micrographs of the CFC-Cu interface region on TS S703 tile 16 after metallographic preparation. The marked area in the larger detail section represents the area analyzed by SIMS. 


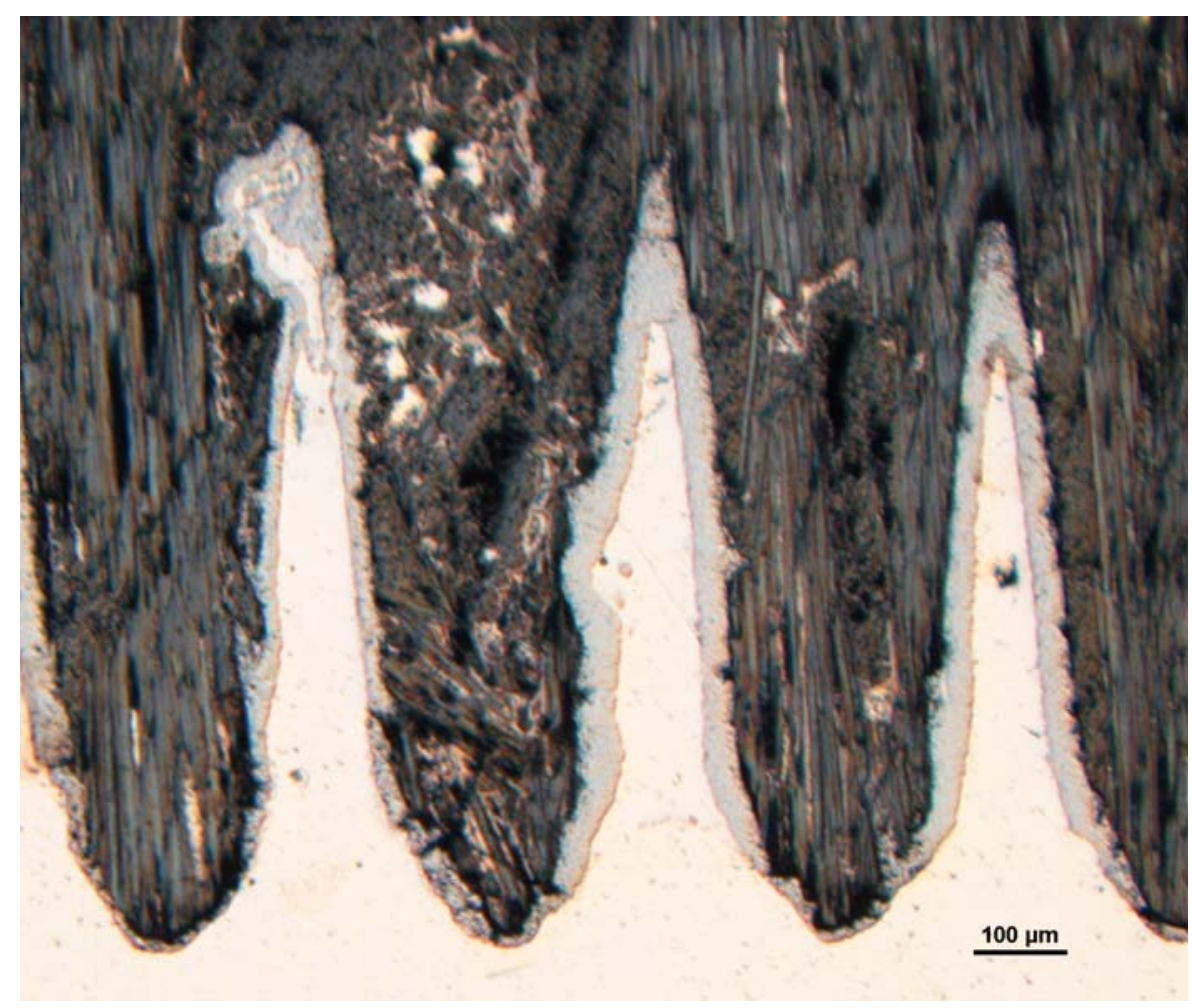

Figure 4 Optical micrographs of a representative area of the CFC-Cu interface region on W7-X tile 4S-045 after metallographic preparation. The AMC solder layer between CFC and $\mathrm{Cu}$ is clearly visible. 

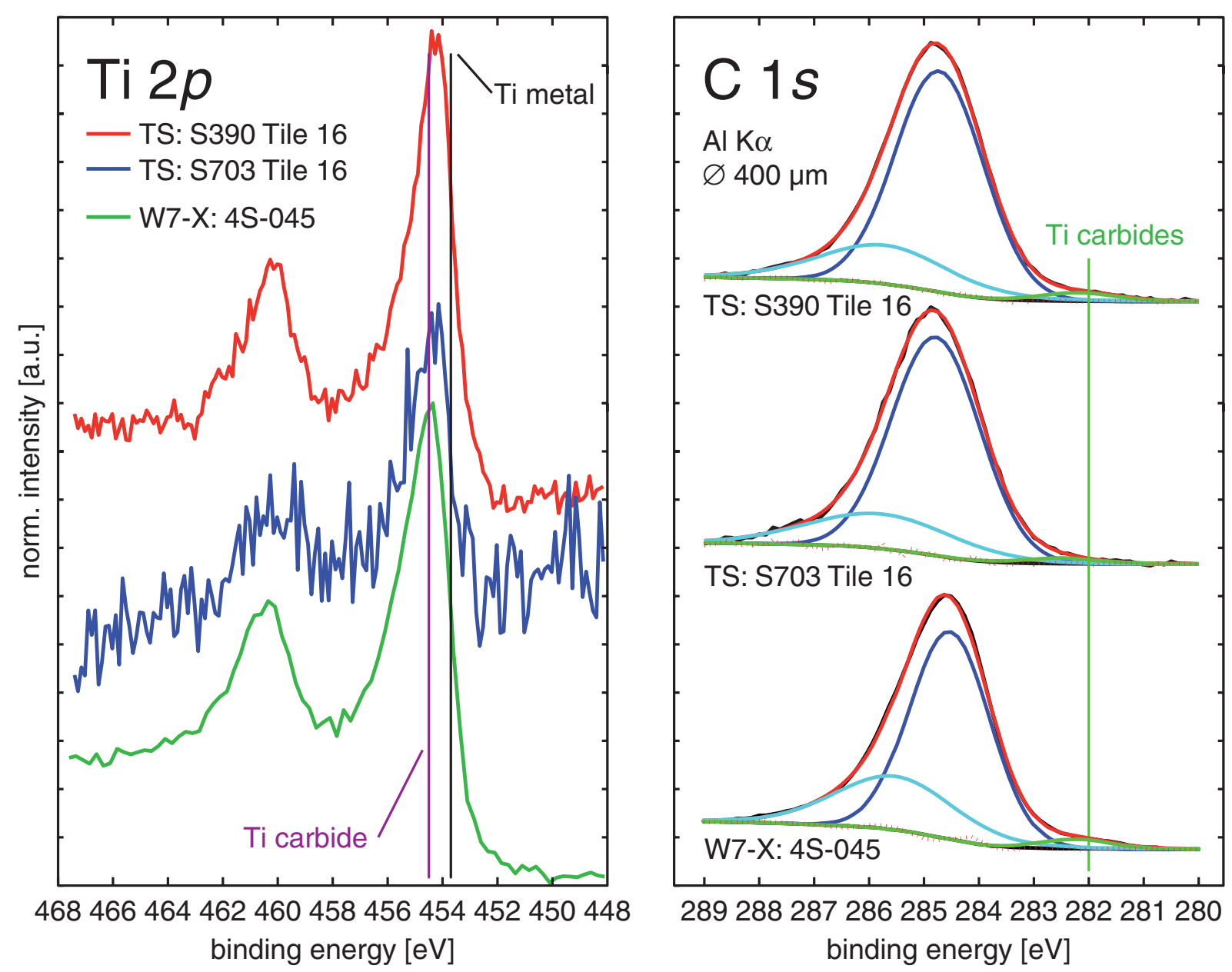

Figure 5 XPS spectra in the CFC-Cu interface regions of the TS and W7-X target elements after heat loading. The Ti $2 p$ regions sow the raw data, the $\mathrm{C} 1 \mathrm{~s}$ regions are fitted with three peaks representing elemental carbon (around 284 and $285 \mathrm{eV}$ ) and carbon bound in carbide (around $282 \mathrm{eV}$ ). 

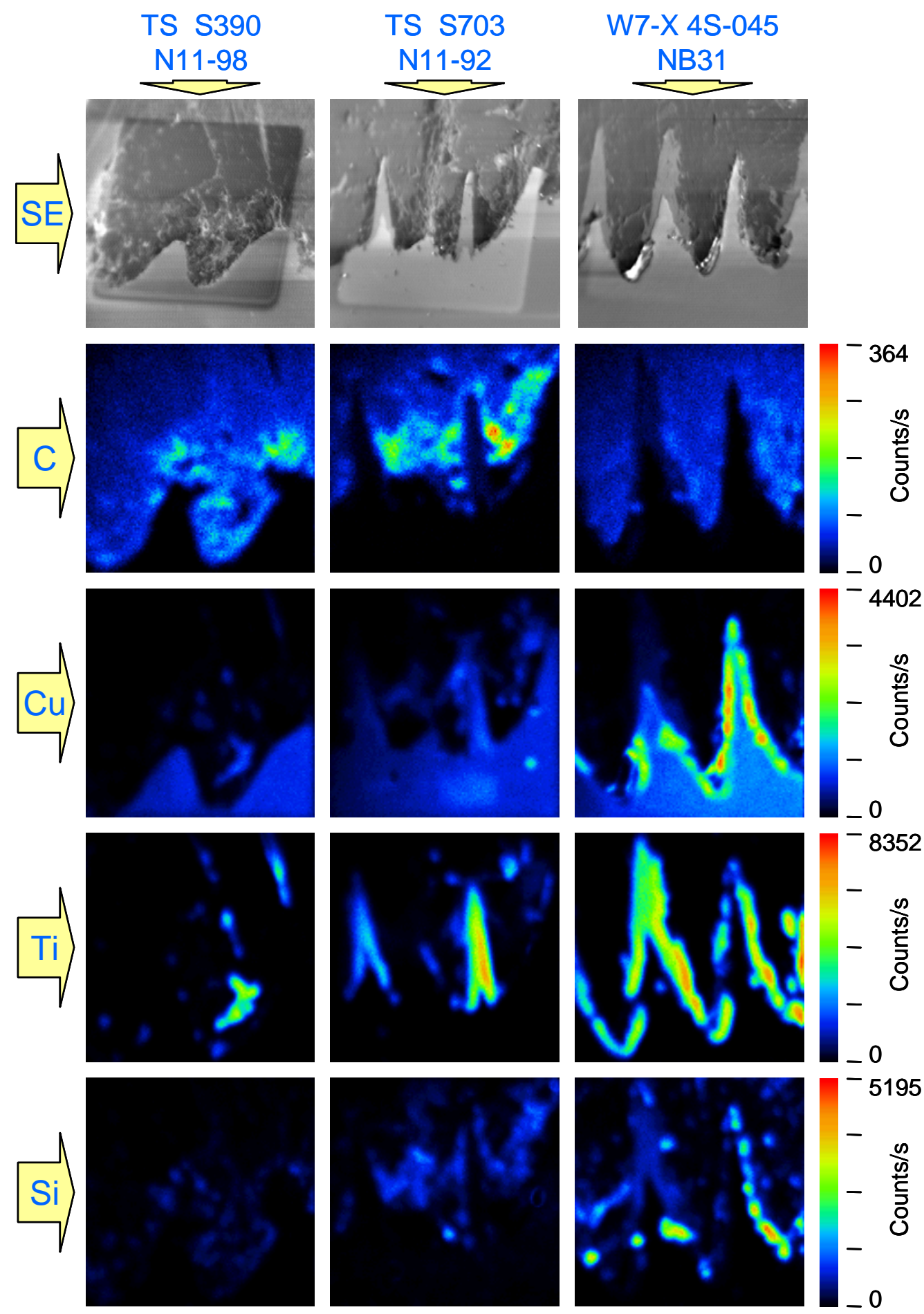

Figure 62 D elemental maps measured by SIMS, representing an area of approximately $1 \times 1 \mathrm{~mm}^{2}$. As the secondary ion yield for different species can vary over orders of magnitude, the numbers given in the color scale are no absolute quantities or can be considered as atomic fractions between the species. However, since the measurement conditions for all three samples were identical, a relative comparison of count rates for the same element on all samples is possible. 